\title{
Vibration Response Prediction of Plate with Particle Dampers Using Cosimulation Method
}

\author{
Dongqiang Wang ${ }^{1}$ and Chengjun $\mathrm{Wu}^{1,2}$ \\ ${ }^{1}$ School of Mechanical Engineering, Xi'an Jiaotong University, No. 28, Xianning West Road, Beilin Discrit, Xian, Shaanxi 710049, China \\ ${ }^{2}$ State Key Laboratory for Strength and Vibration of Mechanical Structures, Xian Jiaotong University, No. 28, \\ Xianning West Road, Beilin Discrit, Xian, Shaanxi 710049, China
}

Correspondence should be addressed to Chengjun Wu; 445wdq@163.com

Received 23 April 2015; Revised 11 July 2015; Accepted 14 July 2015

Academic Editor: Yeesock Kim

Copyright (C) 2015 D. Wang and C. Wu. This is an open access article distributed under the Creative Commons Attribution License, which permits unrestricted use, distribution, and reproduction in any medium, provided the original work is properly cited.

\begin{abstract}
The particle damping technology is a passive vibration control technique. The particle dampers (PDs) as one of the passive damping devices has found wide use in the field of aeronautical engineering, mechanical engineering, and civil engineering because it has several advantages compared with the forms of viscous damping, for example, structure simplicity, low cost, robust properties, and being effective over a wide range of frequencies. In this paper, a novelty simulation method based on multiphase flow theory (MFT) is developed to evaluate the particle damping characteristics using FEM combining DEM with COMSOL Multiphysics. First, the effects of the collisions and friction between the particles are interpreted as an equivalent nonlinear viscous damping based on MFT of gas particle. Next, the contribution of PDs is estimated as equivalent spring-damper system. Then a cantilever rectangular plate treated with PDs is introduced in a finite element model of structure system. Finally frequency response functions (FRFs) of the plate without and with particle dampers are predicted to study characteristics of the particle damping plates under forced vibration. Meanwhile, an experimental verification is performed. Simulation results are in good agreement with experiment date. It is concluded that the simulation method in this paper is valid.
\end{abstract}

\section{Introduction}

Passive control is preferred due to its simplicity and low power consumption. A common passive control device is the particle damper. Particle damping which is a derivative of single-mass impact damper is a promising technique of providing damping with granular particles placed in an enclosure attached to the vibrating structure $[1,2]$. The particle damping can perform well even in severe environments where traditional passive damping methods such as the use of viscoelastic materials are ineffective. Additional benefits of using granular materials instead of a single mass include the elimination of excessive noise and potential damage to the interior wall of the containing hole. The dynamic response of the primary structure is improved by such an additional damping and mass. It offers several advantages due to its conceptual simplicity, potential effectiveness over broad frequency range, temperature and degradation insensitivity, and very low cost [3-8]. In general, metal particles of high density such as lead or tungsten steel are the most common materials for better damping performance.

The particle damper has found wide use in the field of aeronautical engineering, civil engineering, and mechanical engineering [9]. In addition, the particle damping technology has been studied over three decades with a large volume of books and papers in the published literature. However, the modeling of the particle damper remains difficult due to a number of problems. One of the principal reasons in using the particles damper particle is that damping phenomena present remarkable high nonlinear behavior, that is, amplitude dependent. So it is very difficult to design the particle damper to meet the needs in engineering especially for the complex continuum structure [10]. The design of the particle damper is closely related to the large number of parameters, such as the dimension and material of the enclosure, the shape and material of particles, the amount of free space (gap size or volume fraction) given to the particles, the arrangement position of the particle dampers and the level 
of displacement, and acceleration of the primary structure [11].

In order to figure out these issues, most of the modeling efforts have been concentrated in the simplification of the problem where the internal interactions of the particles are not taken into account. For example, a system was studied without any ceiling (the so-called bouncing ball problem) [12]. Many authors modeled particles bed as a single particle [5, 13-16], estimating the performance of the particle damper based on this equivalent particle without considering the collisions and friction effects of the interparticles. Another way to simplify the problem is to linearize the model for different operating conditions. Liu et al. [17] estimated the damping contribution of the particle damper as an equivalent linear viscous damping. Friend and Kinra [5], Bryce et al. $[7,18]$, Chen et al. [1], and Salueña et al. [8] had made much very meaningful research work in the use of the particle dynamics method as referred to discrete element method (DEM). It is very regrettable that the application field is only limited to the single-degree-of-freedom (SDOF) system or the equivalent SDOF system. And the simulation is very time-consuming for computation due to the large number of particles used. If the continuum structure system is subjected to the particle dampers, it is obvious that such analysis will be very complicated. In the practical engineering field, the structure cannot be reasonably well approximated as a SDOF system, since the complex external loading and the interacting particles are likely to excite more than just the fundamental mode of vibration. Although in the early 1990s, the potential of particle damping had been substantiated by the initial testing results of the particle damping [4], there have been some limited numerical and experimental studies on the particle damping $[1,3,5-8,19-21]$. The theory and experimental studies of the continuous particle damping structure are relatively scarce due to the complex interactions involved in particle damping.

Recently, some researchers have performed limited studies to mathematically evaluate the dissipative properties of granular materials using the MFT of gas-particle approach [22]. Wu et al. [22] developed an analytical model of particle damping to evaluate the particle damping characteristics where the interaction effect due to interparticles collisions was quantified as an equivalent viscosity using the MFT of gas particle. Combining this equivalent viscous damping effect with the Coulomb friction damping that expressed all frictional effect, the expression of equivalent drag force for the dynamic analysis of structures integrated with a granular damper was derived. This modeling approach is novelty, since it offers the possibility of capturing the physics nature of granular damping using an analytical perspective with reduced analysis complexity and saving computation time. Fang and Tang [23] further validated the MFT of gasparticle approach based on previous work of $\mathrm{Wu}$ et al. [22] and performed correlated analytical modeling and numerical studies to evaluate qualitatively and quantitatively the energy dissipation in granular damping. Meanwhile, they pointed out the defect in Wu's origin model [22] that the friction effect between the particles was simply expressed as the Coulomb friction damping based on the Hertz contact theory. In order to improve the prediction accuracy of the origin model, $\mathrm{Wu}$ et al. $[24,25]$ further carried out detailed studies on the energy dissipation in particle damping. An improved analytical model for particle damping was developed based on previous work [22], in which the expression of equivalent viscous damping for interparticle frictions was introduced instead of the one of Coulomb friction damping based on the Hertz contact theory in original model. Two typical examples, that is, the free vibration of a cantilever particle damping beam (equivalent SDOF system) and the harmonic forced vibration of a SDOF system with particle damper, were devoted to verify this improved model $[24,25]$. Numerical results showed that the predictions of the improved model agreed well with the experimental results in [22] and the DEM simulations in [23] than that of the original model for appropriate mass packing ratios and excitation levels. However, the above research achievements were limited to the study of a simple system, that is, the SDOF system, and were not applied on the computations of the continuous structure with particle dampers.

The primary objective of this paper is to develop a novelty simulation method based on MFT of gas particle which is capable of rapidly predicting the dynamic response for the complex continuous structure with particle dampers. The software of COMSOL Multiphysics is multiphysics coupling software with powerful processing capacity. It is a flexible platform that allows users to enter coupled systems of partial differential equations (PDEs). The PDEs can be entered directly or using the so-called weak form. Computer simulation has become an essential part of science and engineering. Digital analysis of components, in particular, is important when developing new products or optimizing designs. So the new simulation idea provides a powerful means to analyze the complex continuous structure with particle damper using COMSOL Multiphysics by self-programming.

This paper consists of both theoretical investigation and experimental verification for predicting the characteristics of particle damping. Section 2 details the model development including the mathematical expressions for the equivalent nonlinear viscous damping. In Section 3, numerical study of a cantilever rectangular plate treated with particle dampers is performed. In Section 4, an experimental study is conducted to verify the capacity of this method to predict general characteristics of the particle damping plate. Finally, conclusions are summarized in Section 5.

\section{Model Development}

As mentioned in [26], the granular particles enclosed in a cavity of a vibrating structure can be considered as a multiphase flow of gas particle with low Reynolds number where the particle concentration is high (i.e., the flow is dense). For inelastic particles and a simple shear flow such as a laminar flow, the effective viscosity due to interparticle collisions can be derived from the kinetic theory of dense multiphase flow as follows [26]:

$$
\mu_{c}=\frac{6}{5}\left(1+e_{p}\right) \sqrt{\frac{\Theta}{\pi}} \alpha_{p}^{2} g_{p} \rho_{p} d_{p},
$$


where $\mu_{c}$ is the effective viscosity due to interparticle collisions, $e_{p}$ is the restitution coefficient of the particle, and $\alpha_{p}$ is the packing ratio defined as the volume of particles to the total volume of the cavity. $\rho_{p}$ and $d_{p}$ denote the density and the mean diameter of particles, respectively, $\Theta$ is the fluctuation-specific kinetic energy, and $g_{p}$ is the radial distribution function given by

$$
g_{p}=\frac{1}{1-\alpha_{p}}+\frac{3 \alpha_{p}}{2\left(1-\alpha_{p}\right)^{2}}+\frac{\alpha_{p}{ }^{2}}{2\left(1-\alpha_{p}\right)^{3}} .
$$

The equivalent shear viscosity corresponding to friction force between particles can be expressed as follows [27]:

$$
\mu_{f}=\frac{p_{p} \sin \phi}{2 \sqrt{I_{2 D}}},
$$

where $\phi$ is the angle of internal friction and $I_{2 D}$ is the second invariant of the deviatoric stress tensor. $p_{p}$ is the solids pressure, which is composed of a kinetic term and a second term due to particle collision [28]:

$$
p_{p}=\alpha_{p} \rho_{p} \Theta+2 \rho_{p}\left(1+e_{p}\right) g_{p} \alpha_{p}^{2} \Theta
$$

and the fluctuation-specific kinetic energy is $\Theta=\left\langle\dot{x}^{2}\right\rangle / 3$. For harmonic motion $\left\langle\dot{x}^{2}\right\rangle=|\dot{x}|^{2} / 2$, then (1) can be rewritten as

$$
\begin{aligned}
\mu_{c} & =\frac{6}{5}\left(1+e_{p}\right) \sqrt{\frac{\Theta}{\pi}} \alpha_{p}^{2} g_{p} \rho_{p} d_{p} \\
& =\frac{6}{5}\left(1+e_{p}\right) \sqrt{\frac{|\dot{x}|^{2}}{6 \pi}} \alpha_{p}^{2} g_{p} \rho_{p} d_{p} \\
& =\frac{1}{5}\left(1+e_{p}\right) \sqrt{\frac{6}{\pi}} \alpha_{p}^{2} g_{p} \rho_{p} d_{p}|\dot{x}|
\end{aligned}
$$

with

$$
K_{1}=\frac{1}{5} \sqrt{\frac{6}{\pi}}\left(1+e_{p}\right) \alpha_{p}^{2} g_{p} \rho_{p} d_{p}
$$

where

$$
\mu_{c}=K_{1}|\dot{x}| .
$$

Inserting (4) into (3), then (3) can be rewritten as

$$
\begin{aligned}
\mu_{f} & =\frac{p_{p} \sin \phi}{2 \sqrt{I_{2 D}}} \\
& =\frac{\left(\alpha_{p} \rho_{p}+2 \rho_{p}\left(1+e_{p}\right) g_{p} \alpha_{p}^{2}\right) \sin \phi}{12 \sqrt{I_{2 D}}}|\dot{x}|^{2}
\end{aligned}
$$

with

$$
K_{2}=\frac{\left(\alpha_{p} \rho_{p}+2 \rho_{p}\left(1+e_{p}\right) g_{p} \alpha_{p}^{2}\right) \sin \phi}{12 \sqrt{I_{2 D}}},
$$

where

$$
\mu_{f}=K_{2}|\dot{x}|^{2} .
$$

Considering that the friction model and collision model have the same form of expression, the complete damping effect between the particles can be uniformly expressed as follows:

$$
\mu_{p}=\mu_{c}+\mu_{f}=K_{1}|\dot{x}|+K_{2}|\dot{x}|^{2} .
$$

Furthermore, the viscosity of the gas-particle mixture flow is $\mu_{m}=\mu_{p}+\mu_{g}$. In general, $\mu_{p} \gg \mu_{g}$ and then $\mu_{m} \approx \mu_{p} \cdot \mu_{g}$ is the viscosity of gas. The drag force $F_{d \text {,viscous }}$ of the equivalent viscous damping can be formulated as [29]

$$
F_{d, \text { viscous }}=-\frac{1}{2} \rho_{m} S C_{d}|\dot{x}| \dot{x}=-C_{\text {eq }} \dot{x},
$$

where

$$
C_{\mathrm{eq}}=\frac{1}{2} \rho_{m} S C_{d}|\dot{x}|,
$$

where $C_{\text {eq }}$ represents the equivalent nonlinear viscous damping, $S=d h$ is the cross-section area of the cavity, $d$ is the diameter of the cavity and $h$ is the height of the cavity, $\rho_{m}$ is the equivalent volume density of the mixture flow related to the densities of the gas and the particle, and the drag coefficient $C_{d}$ is given by Sarpkaya [30] where

$$
C_{d}=\frac{f d \pi^{3}}{|\dot{x}|}\left(\frac{3}{2} \beta^{-1 / 2}+\frac{3}{2} \beta^{-1}-\frac{3}{8} \beta^{-3 / 2}\right),
$$

where $\beta=\pi d^{2} f \rho_{m} / \mu_{p}$; inserting (11) into (14), then (14) can be rewritten as

$$
\begin{aligned}
C_{d} & =\frac{f d \pi^{3}}{|\dot{x}|}\left(\frac{3}{2} \beta^{-1 / 2}+\frac{3}{2} \beta^{-1}-\frac{3}{8} \beta^{-3 / 2}\right) \\
& =\frac{f d \pi^{3}}{|\dot{x}|}\left[\frac{3}{2}\left(\frac{\pi d^{2} f \rho_{m}}{\mu_{p}}\right)^{-1 / 2}+\frac{3}{2}\left(\frac{\pi d^{2} f \rho_{m}}{\mu_{p}}\right)^{-1}\right.
\end{aligned}
$$$$
\left.-\frac{3}{8}\left(\frac{\pi d^{2} f \rho_{m}}{\mu_{p}}\right)^{-3 / 2}\right]
$$$$
=\frac{f d \pi^{3}}{|\dot{x}|}\left[\frac{3}{2}\left(\frac{\pi d^{2} f \rho_{m}}{K_{1}|\dot{x}|+K_{2}|\dot{x}|^{2}}\right)^{-1 / 2}\right.
$$$$
+\frac{3}{2}\left(\frac{\pi d^{2} f \rho_{m}}{K_{1}|\dot{x}|+K_{2}|\dot{x}|^{2}}\right)^{-1}
$$

$$
\left.-\frac{3}{8}\left(\frac{\pi d^{2} f \rho_{m}}{K_{1}|\dot{x}|+K_{2}|\dot{x}|^{2}}\right)^{-3 / 2}\right]
$$$$
=\frac{f d \pi^{3}}{|\dot{x}|}\left[\frac{3}{2}\left(\frac{K_{1}|\dot{x}|+K_{2}|\dot{x}|^{2}}{\pi d^{2} f \rho_{m}}\right)^{1 / 2}\right.
$$

$$
+\frac{3}{2}\left(\frac{K_{1}|\dot{x}|+K_{2}|\dot{x}|^{2}}{\pi d^{2} f \rho_{m}}\right)^{1}
$$

$$
\left.-\frac{3}{8}\left(\frac{K_{1}|\dot{x}|+K_{2}|\dot{x}|^{2}}{\pi d^{2} f \rho_{m}}\right)^{3 / 2}\right] .
$$


Inserting (15) into (13), by some mathematical manipulations, one can find that the improved model expression of the equivalent nonlinear viscous damping has a uniform expression due to the interparticles collisions and friction as in the following forms:

$$
\begin{aligned}
& C_{\text {eq }}=\frac{1}{2} \rho_{m} S C_{d}|\dot{x}| \\
&=f \pi^{3} d^{2} h \rho_{m}\left[\frac{3}{4}\left(\frac{K_{1}|\dot{x}|+K_{2}|\dot{x}|^{2}}{\pi d^{2} f \rho_{m}}\right)^{1 / 2}\right. \\
&+\frac{3}{4}\left(\frac{K_{1}|\dot{x}|+K_{2}|\dot{x}|^{2}}{\pi d^{2} f \rho_{m}}\right)^{1} \\
&\left.-\frac{3}{16}\left(\frac{K_{1}|\dot{x}|+K_{2}|\dot{x}|^{2}}{\pi d^{2} f \rho_{m}}\right)^{3 / 2}\right] \\
&=\frac{3 \pi^{3} d^{2} h \rho_{m}}{4}\left(\frac{K_{1}}{\pi d^{2} \rho_{m}}\right)^{1 / 2} f^{1 / 2}|\dot{x}|^{1 / 2} \\
&+\frac{3 \pi^{3} d^{2} h \rho_{m}}{4}\left(\frac{K_{1}}{\pi d^{2} \rho_{m}}\right)|\dot{x}| \\
&+\frac{3 \pi^{3} d^{2} h \rho_{m}}{16}\left(\frac{K_{2}}{\pi d^{2} \rho_{m}}\right)^{3 / 2} f^{-1 / 2}|\dot{x}|^{3} \\
&+\frac{3 \pi^{3} d^{2} h \rho_{m}}{16}\left(\frac{K_{1}}{\pi d^{2} \rho_{m}}\right)^{3 / 2} f^{-1 / 2}|\dot{x}|^{2 / 3} \\
&+ \frac{3 \pi^{3} d^{2} h \rho_{m}}{4}\left(\frac{K_{2}}{\pi d^{2} \rho_{m}}\right)^{1 / 2}|\dot{x}| \\
& \hline d^{2} \rho_{m} \\
&+\left.\dot{x}\right|^{2}
\end{aligned}
$$

where $|\dot{x}|$ is vibration amplitude of vibration velocity and $f$ is the vibration frequency.

It should be noted from (16) that the equivalent viscous damping due to the interparticles frication and collisions for the gas-particle mixture flow in a cavity of a vibrating structure is a kind of high nonlinear damping related to the velocity amplitude of the vibrating structure.

For the continuous particle damping structure, the contribution of particle damper is estimated as an equivalent spring mass system; however, the system does not exhibit any stiffness, that is, mass damping system. The schematic of the particle damper and adopted model are represented in Figure 1. The damping coefficient of the spring mass system is responding to the equivalent nonlinear viscous damping coefficient $C_{\text {eq }}$ (see (16)) determined for different levels of excitation and depending on the excitation velocity amplitude. $M_{\mathrm{eq}}$ represents the mass of the particle damper with the particles. In the simulation, COMSOL Multiphysics software provides simulation option for the spring-massdamper system with access to self-programming. So the equivalent damping due to interparticles collision and friction effects based on the MFT of gas particle is introduced in COMSOL. So the complicated continuous structures treated with the particle dampers are conducted using this equivalent model creatively. Such an idea is novelty and leads to a fire-new breakthrough, since it offers the possibility of predicting the dynamic behavior of a complex continuous structure treated with the particle dampers in a finite element model of a structure with reduced analysis complexity and computational cost. Once the geometric parameters, physical parameters, and boundary conditions of the structure in simulation are set, prediction of the dynamic response of a continuous structure with particle dampers should be implemented by COMSOL. Next, for the sake of brevity, here a simple cantilever rectangular plate with particle dampers is considered as an attempt to validate this method.

A schematic of the considered plate treated with the particle dampers and the adopted model are shown in Figure 2. The plate is modeled by finite element method using discrete Kirchhoff quadrilateral element. The damping contributions of the particle dampers are modeled by the equivalent nonlinear viscous damping dependent on velocity amplitude. Consider the intrinsic structure damping and particle damping; the motion of the global system is governed by

$$
[\mathbf{M}] \cdot\{\ddot{\mathbf{X}}\}+[\mathbf{C}] \cdot\{\dot{\mathbf{X}}\}+[\mathbf{K}] \cdot\{\mathbf{X}\}=\{\mathbf{F}\},
$$

where $\{\mathbf{X}\}$ is the nodal displacement of the plate and $\{\mathbf{F}\}$ is the external force applied to the system. $[\mathbf{K}]$ and $[\mathbf{M}]$ represent, respectively, the stiffness and mass matrix of both the plate and the particle dampers

$$
[\mathbf{M}]=\left[\mathbf{M}_{\mathbf{p}}\right]+\left[\mathbf{M}_{\mathbf{a}}\right],
$$

where $\left[\mathbf{M}_{\mathbf{p}}\right]$ is the mass matrix of the plate and $\left[\mathbf{M}_{\mathbf{a}}\right]$ is the additional mass matrix caused by the presence of the particle damper

$$
\left[\mathbf{M}_{\mathbf{a}}\right]=\left[\begin{array}{cccc}
M_{\mathrm{eq}}^{i} & 0 & \cdots & 0 \\
0 & M_{\mathrm{eq}}^{j} & \cdots & 0 \\
\vdots & & & \\
0 & 0 & \cdots & M_{\mathrm{eq}}^{k}
\end{array}\right]
$$

where $M_{\text {eq }}$ represents the mass of the particle damper with the particles. [C] which represents the damping matrix of the global system is given by

$$
[\mathrm{C}]=\left[\mathrm{C}_{\mathbf{0}}\right]+\left[\mathrm{C}_{\mathrm{eq}}\right] \text {, }
$$

where $\left[\mathbf{C}_{0}\right]$ is the proportional damping matrix of the plate and $\left[\mathbf{C}_{\mathbf{e q}}\right]$ represents the additional damping matrix caused by the particle damper

$$
\left[\mathbf{C}_{\mathrm{eq}}\right]=\left[\begin{array}{cccc}
C_{\mathrm{eq}}^{i} & 0 & \cdots & 0 \\
0 & C_{\mathrm{eq}}^{j} & \cdots & 0 \\
\vdots & & & \\
0 & 0 & \cdots & C_{\mathrm{eq}}^{k}
\end{array}\right] .
$$




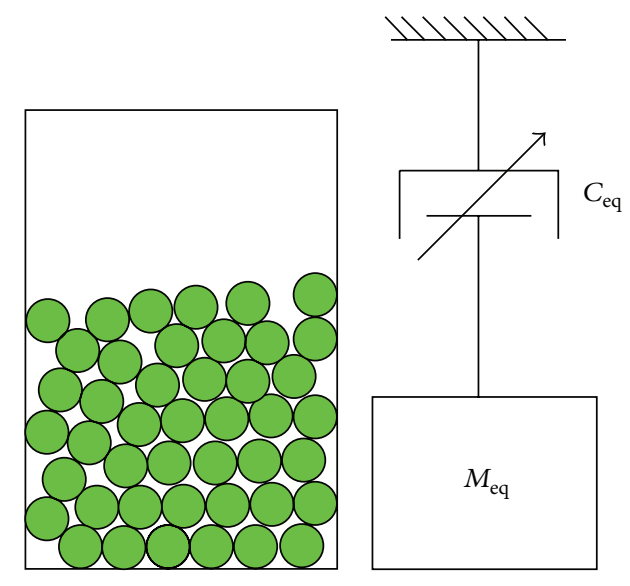

(a)

(b)

FIgURE 1: (a) Sketches of the particle damper and (b) simplification model.

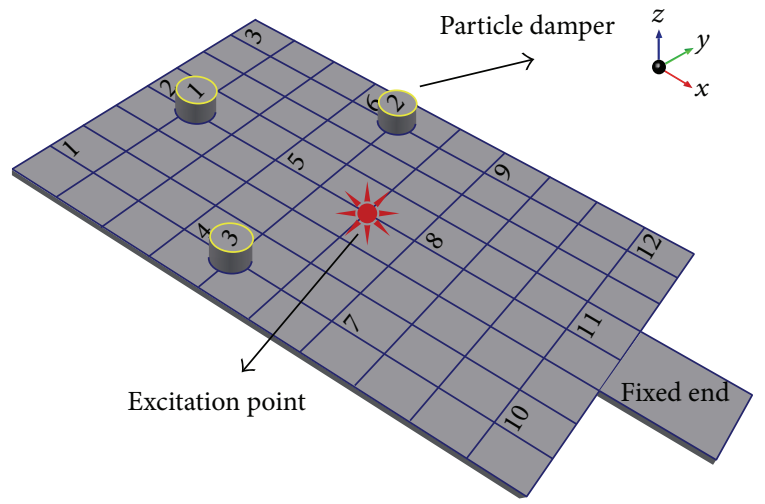

(a)

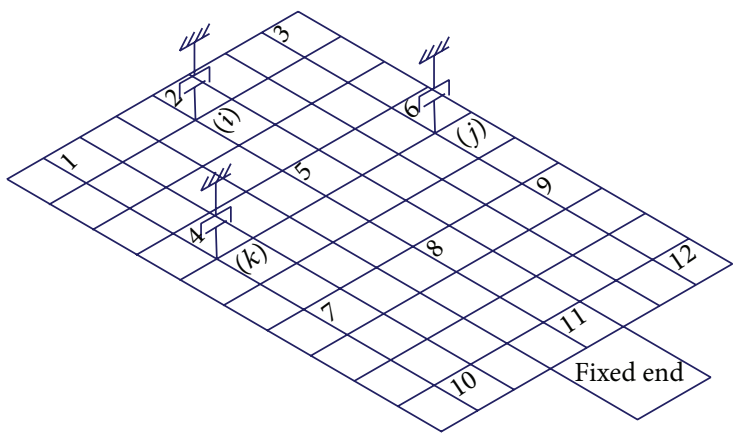

(b)

FIGURE 2: (a) Schematic of plate tread with particle damper and (b) equivalent model adopted.

$C_{\text {eq }}$ represents the equivalent nonlinear viscous damping of each particle damper located at the nodes $i, j$, and $k$ of the plate. One can analyze the responses and the damping characteristics of structures with particle dampers in a finite element model of a structure. The implementation of this modeling is performed in COMSOL environment.

\section{Numerical Simulation Results}

To investigate the performance of the particle damping on a cantilever rectangular plate, the numerical simulation is carried out. The reason why we choose a plate for this study is that it is an infinite DOF system as opposed to the single DOF systems usually studied in the literature $[1,3,5-$ $8,10,31]$. When the structure is excited by a shaker, the structural response could exhibit a large number of modes. This would allow us to investigate the broadband effect of particle damping.

The plate is specified with a mass density $\rho=2646 \mathrm{~kg} / \mathrm{m}^{3}$, Young's modulus $E=5.6 \times 10^{10} \mathrm{~Pa}$, and Poisson ratio $v=0.27$. The plate dimensions are length $L=300 \mathrm{~mm}$, width $W=200 \mathrm{~mm}$, and thickness $e=6 \mathrm{~mm}$. The mass of the enclosure is $14.52 \mathrm{~g}$ and its interior diameter and height are $16 \mathrm{~mm}$ and $20 \mathrm{~mm}$, respectively. The particle is made of tungsten powder whose density is $17000 \mathrm{~kg} / \mathrm{m}^{3}$, and the mean diameter of particles is $0.3 \mathrm{~mm}$. The restitution coefficient of particles is 0.6 on the basis of testing. The kinetic friction coefficient between the particles is 0.3 from experimental results. In addition, the kinematic viscosity and density of air are $1.51 \times 10^{-5} \mathrm{~m}^{2} / \mathrm{s}$ and $1.21 \mathrm{~kg} / \mathrm{m}^{3}$, respectively.

The arrangement location of three particle dampers and the excitation point are indicated in Figure 2. The three particle dampers used in this test have the same design parameters. This experiment is tested with the same mass of particles $\left(\alpha_{\mathrm{mp}}=40 \%\right)$ in each particle damper. The particles mass of each particle damper filled is $11.60 \times 10^{-3} \mathrm{~kg}$.

Here, a term named mass packing ratio (denoted by $\alpha_{\mathrm{mp}}$ ) is introduced for the sake of the convenience for experimental verification. It should be noted that $\alpha_{\mathrm{mp}}$ is different from the packing ratio $\alpha_{p}[22,23,32]$ (i.e., $\alpha_{p}=0.63 \alpha_{\mathrm{mp}}$ ). The mass packing ratio is defined as the actual packing mass of particles to the maximum permissive packing mass of particles in a cavity. 

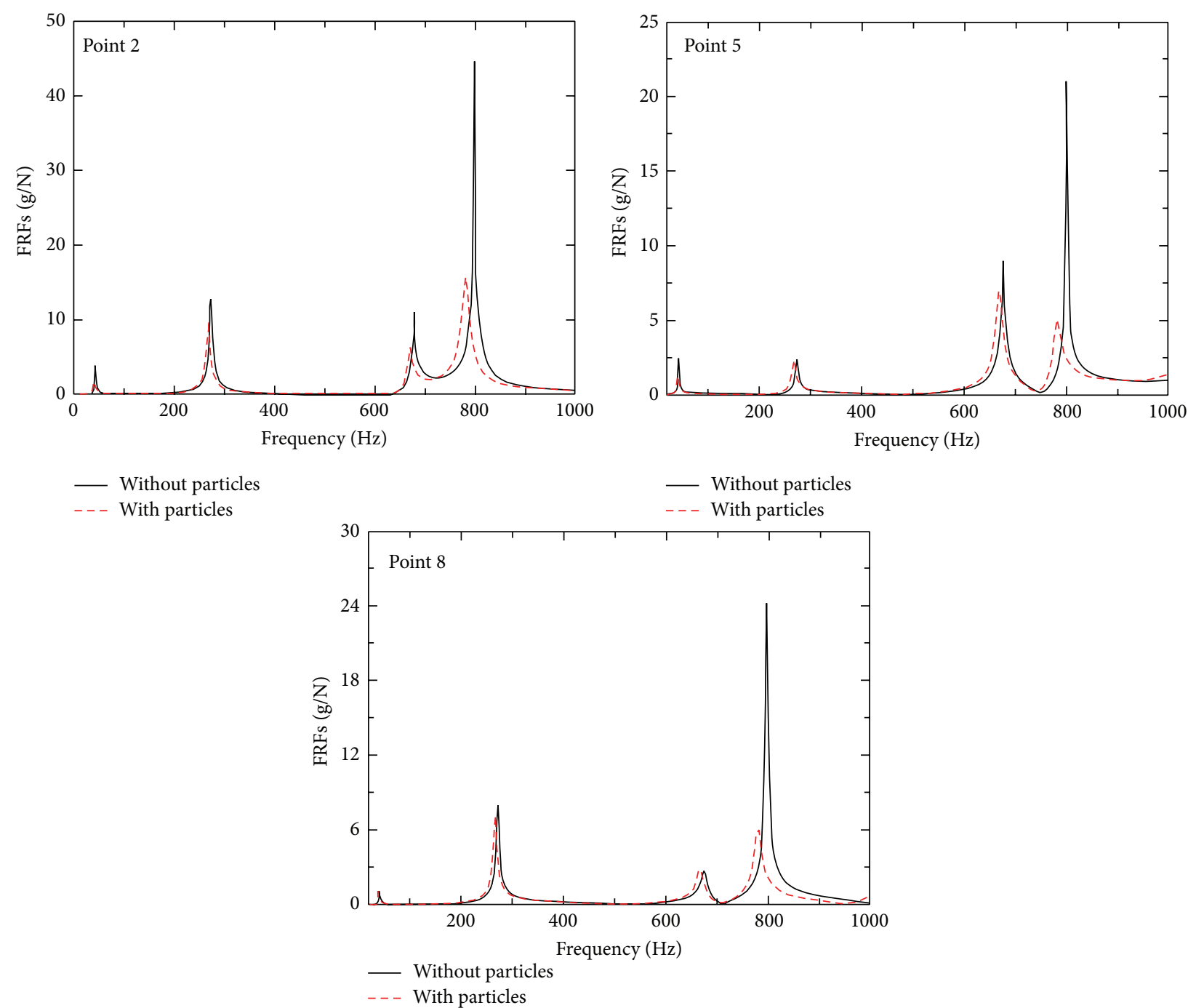

FIGURE 3: The simulation result comparison of the FRFs on the rectangular plate for the case without and with particle dampers.

In order to identify the damping of the particle damper, the evolutions of both force and acceleration of the system versus the frequency of excitation are measured. The frequency response functions (FRFs) acceleration/force of the plate are successively measured at twelve points of the plate. A broadband random excitation is applied to the rectangular plate with a maximum frequency of $1000 \mathrm{~Hz}$, for the solid rectangular plate without particles damper and with three particle dampers, respectively. A sine-sweep excitation is used with a small frequency step.

To validate the finite element model, the first four natural frequencies of the system formed by the plate without particle dampers are compared with those of the experiment. The natural frequencies of first four bending modes tested in the experiment along the $Z$ direction are, respectively, $40.74 \mathrm{~Hz}$, $263.29 \mathrm{~Hz}, 653.53 \mathrm{~Hz}$, and $828.25 \mathrm{~Hz}$. The natural frequencies of first four bending modes along the $Z$ direction are analyzed using FEM analysis by COMSOL which are $39.06 \mathrm{~Hz}$, $270.31 \mathrm{~Hz}, 685.94 \mathrm{~Hz}$, and $814.06 \mathrm{~Hz}$, respectively. There are relatively small changes in the first four natural frequencies by comparing simulation results and experiment date. The variations do not exceed $4.72 \%$ which shows a good agreement between the experiment date and the simulation results. The goal of this set of tests is to verify geometric model, load, and boundary condition applied; this also includes the analysis and calibration of the experiment parameters, which have an effect on the measurement precision.

To get the structural damping of the plate considered in the theoretical model, a narrow-band random excitation is applied on the cantilever rectangular plate. Then, the motility half-power bandwidth method is applied to measure the damping ratio for a specified measurement point. Namely, $\zeta=\Delta \omega / 2 \omega_{n}$ is similar to the approach taken in $[3,6]$.

Figure 3 shows that numerical simulations of the FRFs (acceleration/force) of the undamped plate (without particle dampers) and the plate with the particle dampers are calculated at any arbitrary three points, respectively. They are points 2, 5, and 8 as shown in Figure 2. The effectiveness of the particle dampers for reducing the vibration levels of the structure over a wide frequency band is shown in Figure 3 


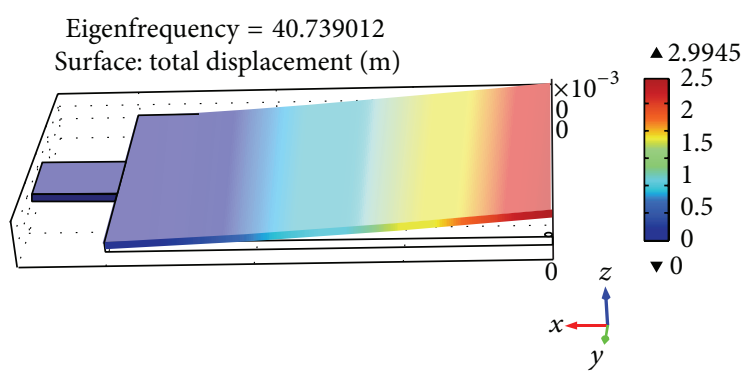

FIGURE 4: Vibration mode shape of the cantilever rectangular plate without particle dampers under the first order of natural frequency.

by the examinations of these FRFs (the plate without and with particle dampers). The effects of the particle dampers are visible on each one of the first four modes of the plate. It is found that the presence of particle dampers causes an increase of modal damping which can reach quite high levels without significant changes of the natural frequencies and mode shapes compared with the case of the plate without particle dampers. The results show that the particle damping is remarkably effective, and the strong attenuations are achieved within a broad frequency range for achieving high damping effect from the use of a minimal quantity of particles. The rate of the total particles mass $(34.8 \mathrm{~g})$ to the primary structure mass $(1 \mathrm{~kg})$ is only $3.5 \%$.

It is noted in Figure 3 that frequencies shifting happens in the FRFs curves when the particle dampers are exerted on the plates comparing with the case of the plate without particle dampers. The reason is that the particle dampers added change the mass matrix of the whole system. As a consequence, the inherent frequency of each order modal is reduced when the particle dampers are exerted on the plates.

It is noteworthy that we can see from Figure 3 that the vibration mode around $800 \mathrm{~Hz}$ seems to be more energetic than the fundamental mode. Through the analysis of the eigen frequencies of the cantilever rectangular plate without particle dampers, the first and fourth orders of natural frequencies along the $Z$ direction are got; they are $40.74 \mathrm{~Hz}$ and $828.25 \mathrm{~Hz}$. The longitudinal vibration mode shapes of the first and fourth orders of natural frequencies are shown in Figures 4 and 5 .

We can get by the analysis of modal frequencies and mode shapes that it shows that the acceleration amplitudes of vibration of the rectangular plate at measuring points 2,5 , and 8 are higher than the case of the first natural frequency from range value of color legend in Figures 4 and 5 when the response frequency is equal to the fourth natural frequency. For a better view of the comparison, the data of the acceleration amplitude is retrieved from the same observation point on the rectangular plate without particle dampers in the different natural frequencies. It is quite clear that the vibration mode in the fourth order of natural frequency seems to be more energetic than the fundamental mode (see Figure 6). It also demonstrates that the vibration amplitude at measurement point is also related to the mode shapes. Similar phenomenon can also be seen from Figure 3

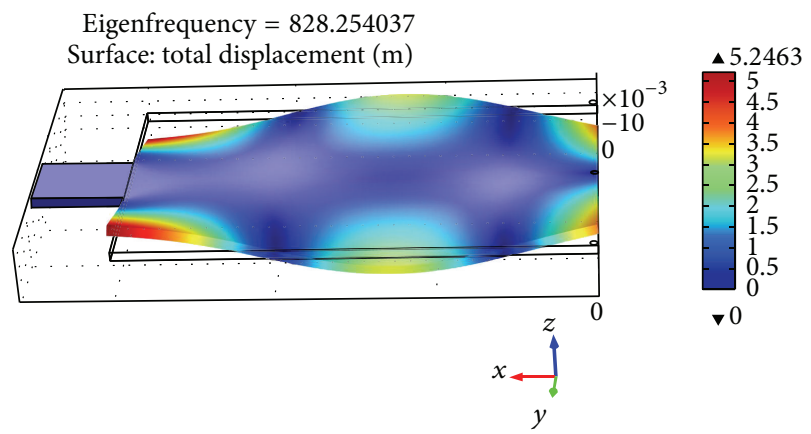

FIGURE 5: Vibration mode shape of the cantilever rectangular plate without particle dampers under the fourth order of natural frequency.

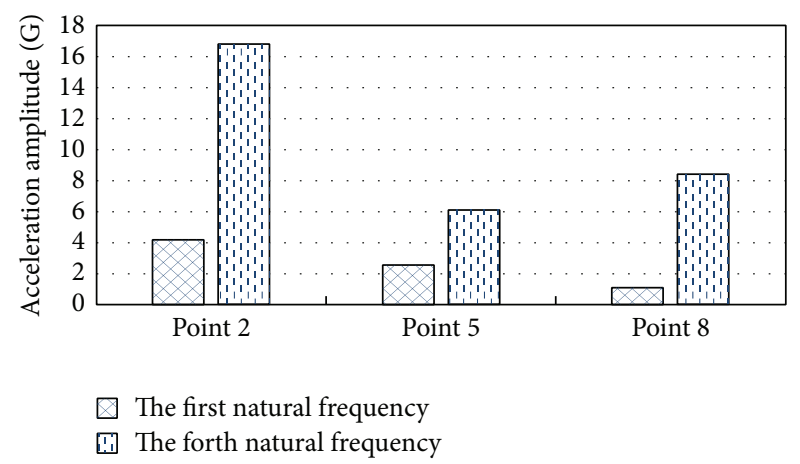

FIGURE 6: The comparison of the acceleration amplitude at the same observation point on the rectangular plate under the different natural frequencies.

when the plate is with particle dampers, which may be originated from the same reason.

It is worth mentioning in Figure 3 that particle dampers are known to have a tremendous potential to provide vibration suppression comparing with the case without particle dampers over wide frequency band from 0 to $1000 \mathrm{~Hz}$. In a number of resonance frequencies, the particle damping exhibits reduction of the response amplitude to some extent. We observe that the damping performance of the particles is more remarkable from $600 \mathrm{~Hz}$ to $1000 \mathrm{~Hz}$ than the case from 


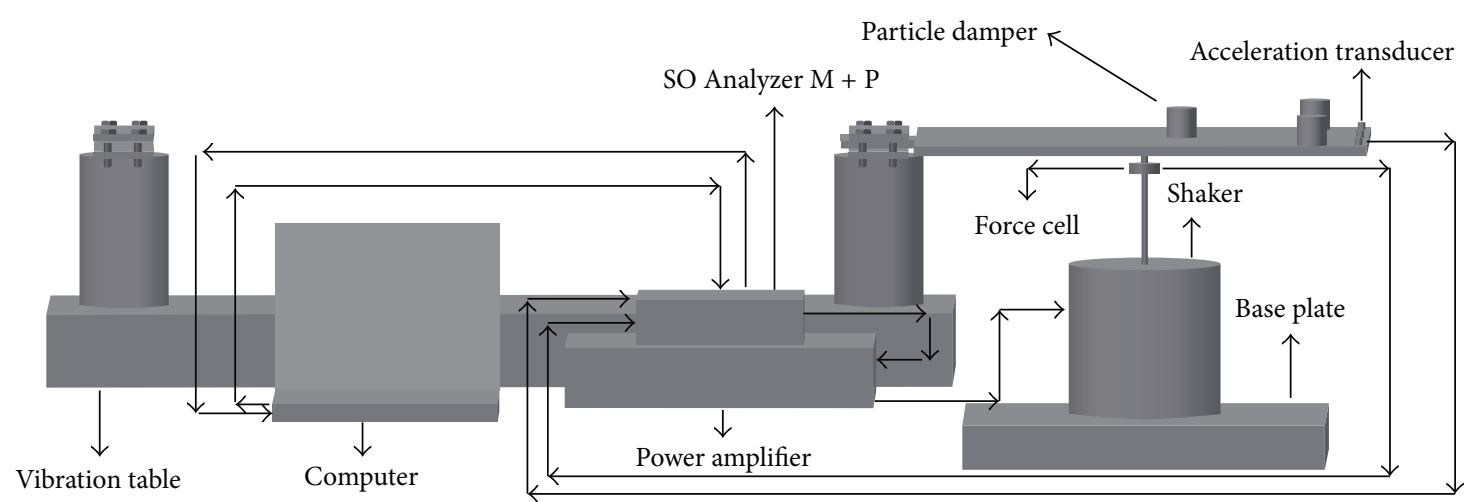

FIGURE 7: Schematic diagram of experimental setup.

0 to $600 \mathrm{~Hz}$. This also reveals that the damper efficiency is more prominent for high modes. The phenomenon mainly stems from the fact that the most commonly applied passive vibration control techniques are based on the mass-springdamping system. One of the most important features in forced vibration is that the passive control effect is significant when the forcing frequency is greater than the natural frequency $\left(\omega / \omega_{0}>\sqrt{2}\right)$. In other words, the damping efficiency of the particle damper is more prominent for high modes. To be honest, this is a shortcoming of particle damping technology. Other studies have come to similar conclusions $[11,33,34]$.

In a word, the particle dampers have good performance in reducing the response of structures under dynamic loads. Such a strong damping effect has been consistently observed in each measurement point. In the next section, an experimental verification is performed to illustrate the accuracy of the simulation results and evaluate the theoretical model.

\section{Experimental Validation}

To verify the simulation method developed in this study, an experiment for a cantilever rectangular plate with three particle dampers is set up and shown in Figure 7. The specifications for the experiment are the same as that used in the simulation for the purpose of comparison. The experimental process is organized in two parts. In the first part, the cantilever rectangular plate without particle damper is first tested in order to characterize the modal behavior of the primary structure. In the second part, the measurement is repeated with the three particle dampers in order to reveal the impact of the particle dampers and describe the dynamic behavior of the particle damping structure in the considered frequency band.

A schematic of the experimental apparatus is shown in Figure 8. The experimental model consists of the primary structure (cantilever plate) and three aluminum enclosures containing tungsten particles. The enclosures that are partially filled with tungsten particles $\left(\alpha_{\mathrm{mp}}=40 \%\right)$ are attached to the plate which is itself attached to an electromagnetic

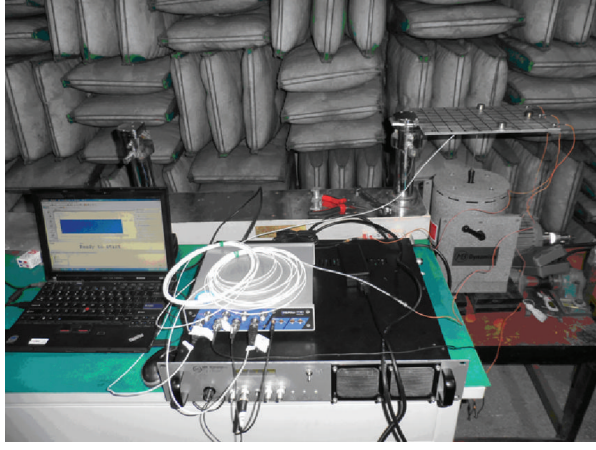

Figure 8: A picture of the experimental apparatus used.

shaker (M B MODAL 50A). The shaker provides the excitation force. The signal of the harmonic excitation amplified by power amplifier (M B500VI) is transferred to the shaker. The force and acceleration signals are measured with the force transducer (Dytran 1051V4) and acceleration transducer (Dytran 3133B1) having a mass of $0.6 \mathrm{~g}$, respectively. A Dynamic Signal Analyzer (M+P SO Analyzer) is used to collect and process the data. Then the measurements are carried out successively at twelve points distributed on the rectangular plate as shown in Figure 2. The frequency response functions (FRFs) acceleration/force of the rectangular plate are successively measured at twelve points of the plate rectangular by moving the acceleration transducer at each location. For each measurement, a stepped-sine excitation generated by $\mathrm{M}+\mathrm{P}$ SO Analyzer is amplified and then input into the shaker. A predefined level of force is chosen and maintained throughout the test thanks to a closed-loop control by M B 500VI. After the measurement, the modal characteristics are identified from the twelve FRFs using the software $\mathrm{M}+\mathrm{P}$ SO Analyzer which is developed by $\mathrm{M}+\mathrm{P}$ International $\mathrm{GmbH}$ in Germany.

Figure 9 presents comparison of the FRFs (acceleration/force) of the cantilever rectangular plate without particle dampers between the simulation results and experiment date. They are, respectively, calculated at points 2,5 , and 8 as shown 

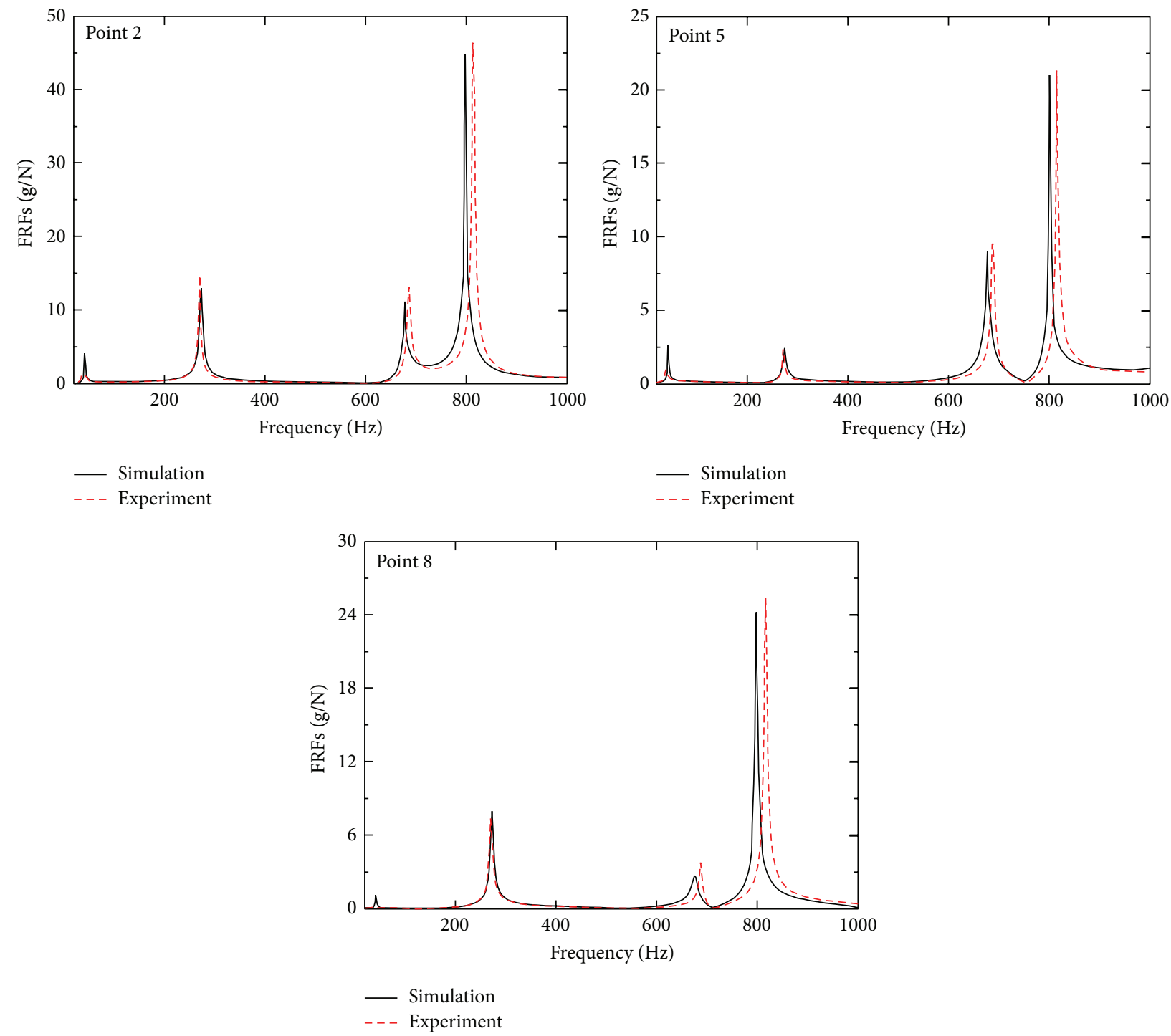

FIGURE 9: The comparison of the FRFs on the rectangular plate between simulation and experiment for the case without particle dampers.

in Figure 2. It is noted that the simulation results in COMSOL differ slightly from the experimental results. That is to say, the structural damping considered in the simulation model is not exactly the actual intrinsic structural damping of the plate in the experiment. Again, a similar trend is observed in the results as shown in Figure 9 for harmonic excitation at points 5 and 8 , respectively. The peak in the FRFs obviously shifts toward the left in the simulation results comparing with the case of the experiment date. In this case, such shift phenomenon is possibly due to the connection type between the rectangular plate and the electromagnetic shaker (see Figure 8), which are connected by adhesive. It is obvious that the adhesive is an additional constraint condition for boundary conditions of the rectangular plate, which leads to natural frequencies shift.

Figure 10 presents comparison of the FRFs (acceleration/force) of the cantilever rectangular plate with particle dampers between the simulation results and experiment date.
They are, respectively, calculated at points 2,5 , and 8 as shown in Figure 2. Observing this figure, the acceleration responses between the theoretical results and experimental data agree well in general. There are also differences at the peak amplitudes in the vicinity of the natural frequencies between the numerical and experimental responses for the system with particle dampers. These differences stem from the hypothesis considered when modeling the system. Nevertheless, the simulation results show the ability of the model developed in this work to predict the dynamic behavior of the structure taking into account the effect of particle damping for wide frequency range.

In order to further verify the model's applicability for various kinds of conditions, the typical behaviors of the system with particle dampers, presented as frequency response functions, are shown in Figures 11-13, which correspond to three different cases (mass ratio, particle material, and particle size). The first case is that the mass ration of 

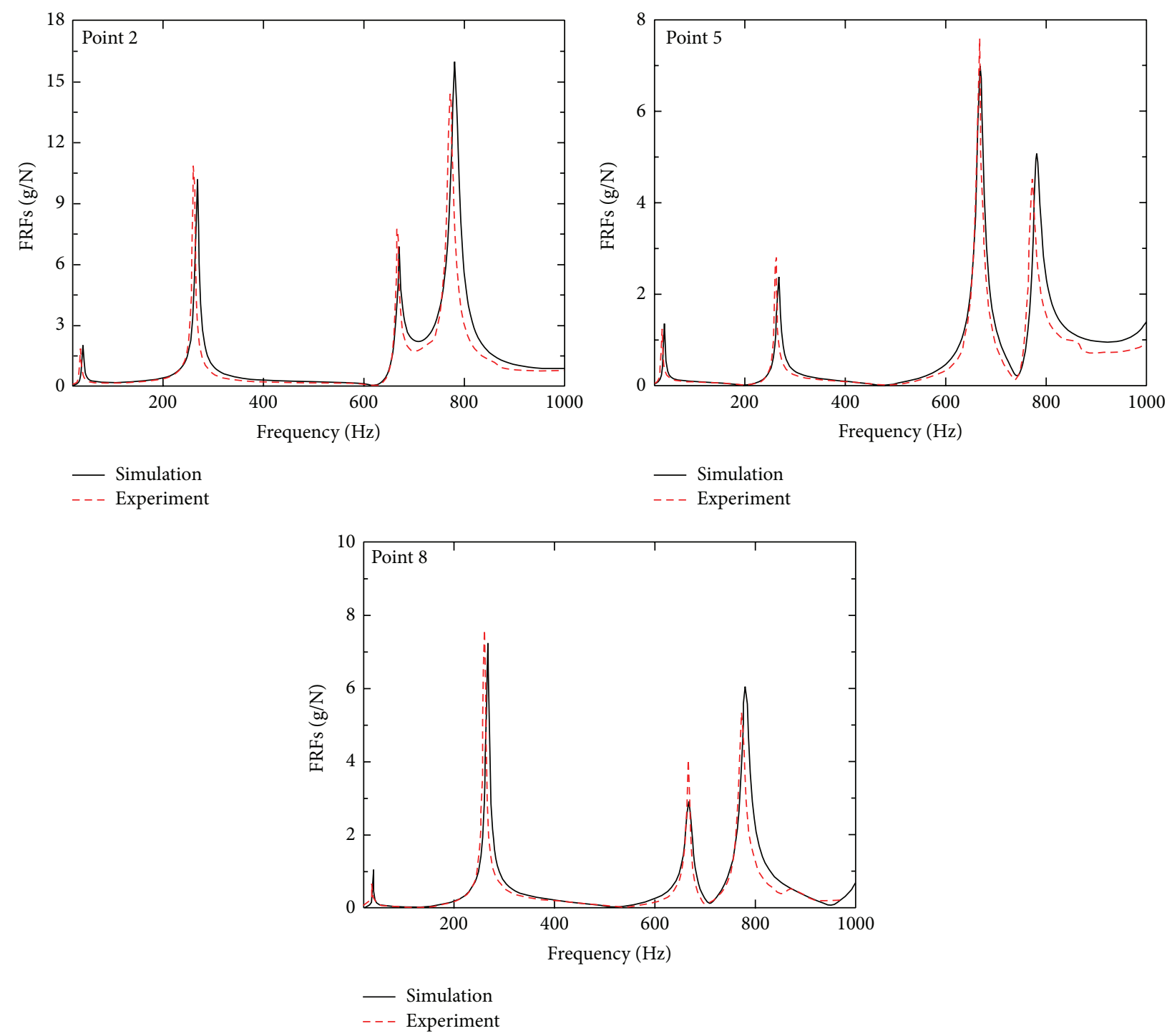

FIGURE 10: The comparison of the FRFs on the rectangular plate between simulation and experiment for the case with particle dampers.

the enclosures filled with tungsten particles changes to $70 \%$ and the other parameters remain the same; the second case is that steel balls of rolling bearing are used as filled material and the mass ratio is also $40 \%$; the third case is that iron powder is substituted for tungsten powder. The particle dampers arrangement, the measurement points, and exciting point still hold the same as those listed above in this paper. The steel balls of rolling bearing and iron powder are chosen because they are readily available. Physical parameters of the steel balls of rolling bearing and iron powder are density: $7734 \mathrm{~kg} / \mathrm{m}^{3}$; restitution coefficient: 0.75 ; diameter: $1 \mathrm{~mm}$ and density: $6800 \mathrm{~kg} / \mathrm{m}^{3}$; restitution coefficient: 0.6 ; diameter: $0.3 \mathrm{~mm}$, respectively.

In Figures 11-13, the responses predicted by the analytical model proposed in this research are simulated over the broadband frequency band, and the responses at a selective point are then calculated for verification purpose. It can be observed that, for all cases considered, the analytical predictions have excellent agreement with the direct experiments. This clearly demonstrates the validity of the proposed analytical model. These analysis results show that the granular damping has considerable vibration suppression capability, especially for higher-order modes.

For cavity size, this case is not a further validation. Change of cavity size means mass packing change. Simonian thinks that, as far as forced vibration to be concerned, the resulting damping performance depends on vibration amplitude and mass packing ratio [6]. In forced vibration applications, there is an optimal mass packing ratio for a given vibration amplitude. Further work is needed to analytically and experimentally model their behavioral characteristics for further developments in particle damper technology in our next work.

Due to many subjective and objective reasons, the error definitely exists between simulation and experiment results. Firstly, we ignore the interactions between particles and 

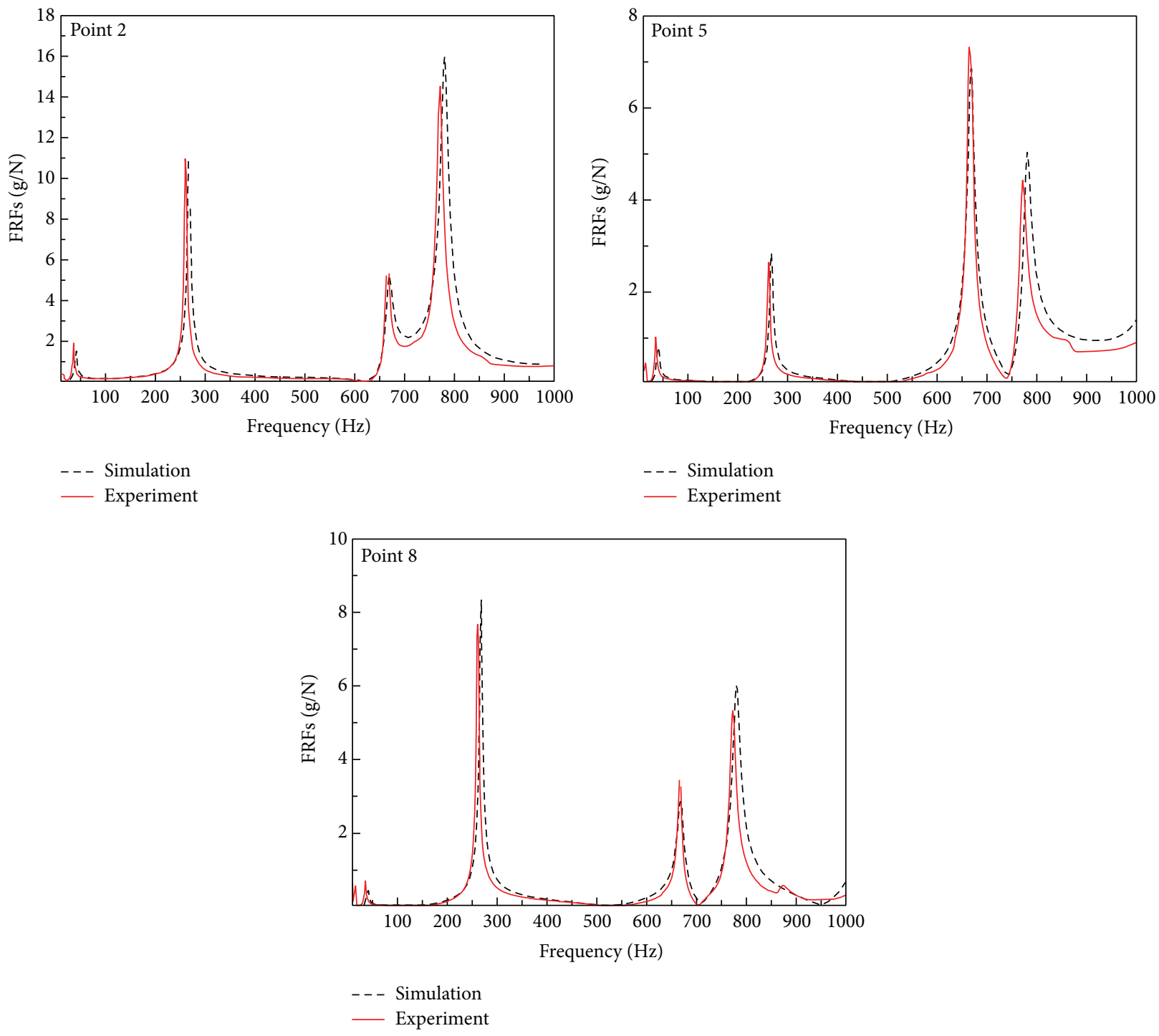

FIGURE 11: The comparison of the FRFs on the rectangular plate between simulation and experiment for the case with particle dampers (mass packing, $70 \%$ ).

the wall of the enclosure and we use series of numerical methods to obtain approximate solutions. Therefore, the theoretical model is not a complete description for particle damping but an approximate expression, which causes the error. Secondly, the exciter is attached to the plates in the experiment process, while this constraint cannot be reflected in the process of simulation. Thirdly, every condition and material property are ideal in the simulation but the plates we use in the experiment are not made of uniform aluminum alloy sheet and it is hard to acquire the actual value of Young's modulus, Poisson's ratio, and structural damping coefficient. As a result, the accuracy of simulation is affected by abovementioned factors. Although there are unavoidable errors, they are still in tolerance range. Therefore, the results of the comparison between the experiment date and simulation results are convincing to prove the validity and reliability of the theoretical model.
Generally speaking, the theoretical model based on multiphase flow theory of gas particle is efficient for estimating the vibration response of the particle damping plate with good accuracy and reliability. Comparing with the DEM simulation, this theoretical model is less time-consuming and easier for calculating with wider applicability than the DEM method.

\section{Concluding Remarks}

In this paper, a novelty simulation method based on twophase flow theory is developed to evaluate the damping characteristics for the continuum structure with particle dampers using finite element method combining discrete element method by COMSOL Multiphysics. In this work, the effect of the collisions and friction between the particles is interpreted as an equivalent nonlinear viscous damping based 

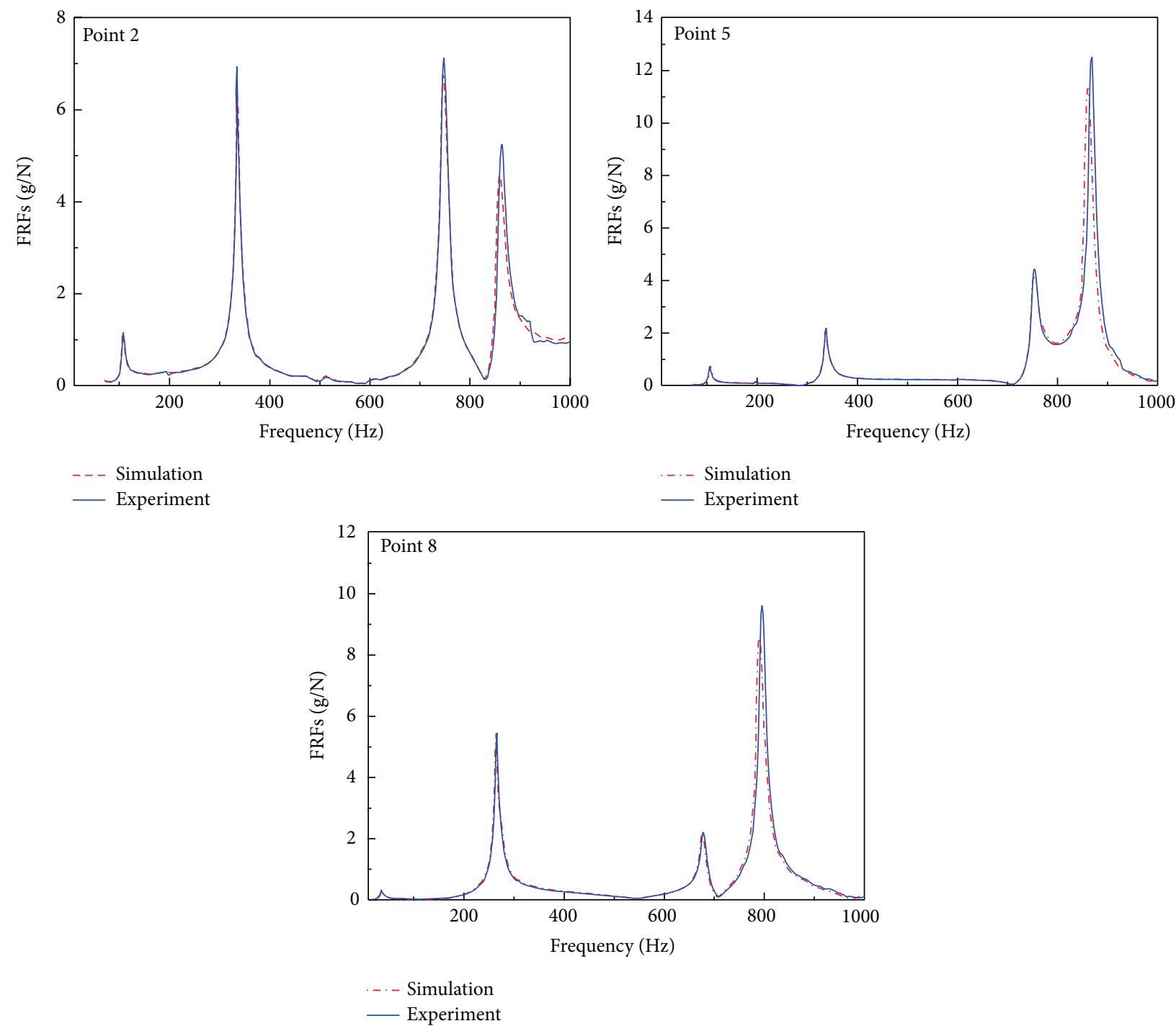

FIGURE 12: The comparison of the FRFs on the rectangular plate between simulation and experiment for the case with particle dampers (filled with steel balls of rolling bearing).

on two-phase flow theory of gas particle to characterize the damping of the particles dampers. Such an idea is novelty and leads to a fire-new breakthrough, since it offers the possibility of capturing the physics nature of granular damping using an analytical perspective to predict the dynamic behavior of a complex continuous structure treated with the particle dampers in a finite element model of a structure with reduced analysis complexity and computational cost. The dynamic responses of a plate treated with the particle dampers under harmonic excitation are predicted. An experiment is performed, and a good agreement between the model prediction results and experimental results shows that this simulation method in this paper is valid. The experimental verifications prove that the particle damping is remarkably effective, and strong attenuations are achieved within a broad frequency range. It would facilitate the development of application techniques for achieving high damping effect by the use of a minimal quantity of particles. As expected, changes in the total particle mass can lead to a fairly significant shift in the frequency of peak response.

This simulation method provides an effective instruction to the implementation of particle damping in practice and offers the possibility of analyzing more complex particle damping system with lower computational cost than DEM. And it can lay a theoretical foundation for solving the vibration and acoustic radiation response prediction problem of particle damping composite structures. It is noted that the entire model has higher prediction accuracy and provides convenience for further studies in depth.

\section{Conflict of Interests}

The authors declare that there is no conflict of interests regarding the publication of this paper. 

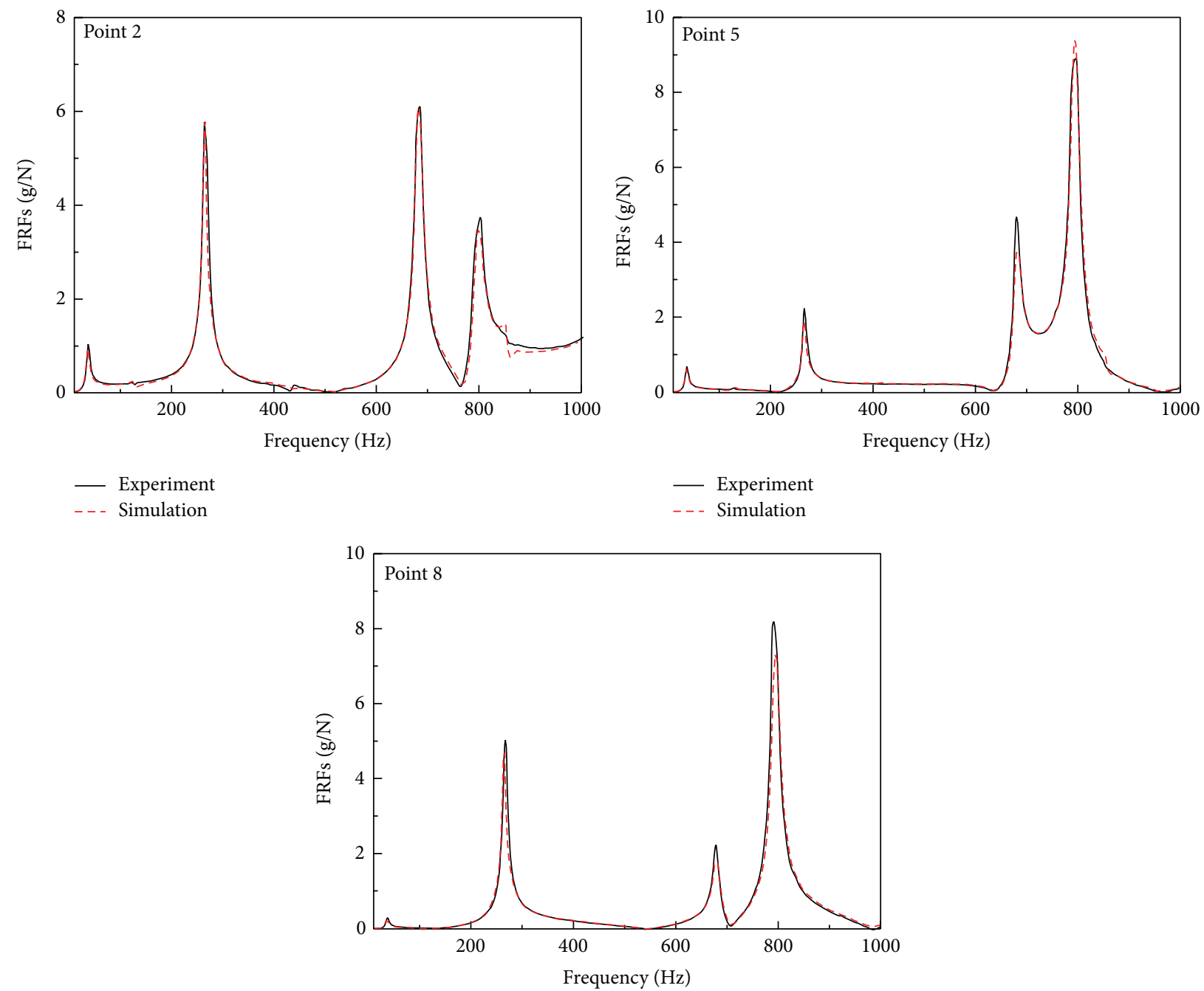

- Experiment

- - - Simulation

FIGURE 13: The comparison of the FRFs on the rectangular plate between simulation and experiment for the case with particle dampers (filled with iron powder).

\section{Acknowledgments}

The work described in this paper was supported by the Natural Science Foundation of China (NSFC) (no. 51075316) and Program for Changjiang Scholars and Innovative Research Team in University (PCSIRT) (no. IRT1172).

\section{References}

[1] T. Chen, K. Mao, and X. Huang, "Dissipation mechanisms of non-obstructive particle damping using discrete element method," in Smart Structures and Materials 2001: Damping and Isolation, vol. 4331 of Proceedings of SPIE, pp. 294-301, Newport, Calif, USA, March 2001.

[2] J. R. Fricke, "Lodengraf damping: an advanced vibration damping technology," SV Sound and Vibration, vol. 34, no. 7, pp. 2227,2000 .
[3] J. J. Hollkamp and R. W. Gordon, "Experiments with particle damping," in Smart Structures and Materials: Passive Damping and Isolation, vol. 3327 of Proceedings of SPIE, pp. 2-12, International Society for Optics and Photonics, San Diego, Calif, USA, March 1998.

[4] H. V. Panossian, "Structural damping enhancement via nonobstructive particle damping technique," Journal of Vibration and Acoustics, vol. 114, no. 1, pp. 101-105, 1992.

[5] R. D. Friend and V. K. Kinra, "Particle impact damping," Journal of Sound and Vibration, vol. 233, no. 1, pp. 93-118, 2000.

[6] S. S. Simonian, "Particle beam damper," in Smart Structures and Materials 1995: Passive Damping, Proceedings of SPIE, pp. 149160, San Diego, Calif, USA, May 1995.

[7] L. F. Bryce, M. F. Eric, and E. O. Steven, "Effectiveness and predictability of particle damping," in The 7th Annual International Symposium on Smart Structures and Materials, vol. 3989 of Proceedings of SPIE, International Society for Optics and Photonics, Newport Beach, Calif, USA, 2000. 
[8] C. Salueña, T. Pöschel, and S. E. Esipov, "Dissipative properties of vibrated granular materials," Physical Review E, vol. 59, no. 4, pp. 4422-4425, 1999.

[9] H. V. Panossian, "Non-obstructive particle damping: new experiences and capabilities," in Proceedings of the 49th AIAA/ASME/ASCE/AHS/ASC Structure, Structural Dynamics and Materials Conference, Schaumburg, Ill, USA, April 2008.

[10] A. Papalou and S. F. Masri, "An experimental investigation of particle dampers under harmonic excitation," Journal of Vibration and Control, vol. 4, no. 4, pp. 361-379, 1998.

[11] M. Trigui, E. Foltete, and N. Bouhaddi, "Prediction of the dynamic response of a plate treated by particle impact damper," Proceedings of the Institution of Mechanical Engineers, Part C: Journal of Mechanical Engineering Science, vol. 228, no. 5, pp. 799-814, 2014.

[12] P. J. Holmes, "The dynamics of repeated impacts with a sinusoidally vibrating table," Journal of Sound and Vibration, vol. 84, no. 2, pp. 173-189, 1982.

[13] K. S. Marhadi and V. K. Kinra, "Particle impact damping: effect of mass ratio, material, and shape," Journal of Sound and Vibration, vol. 283, no. 1-2, pp. 433-448, 2005.

[14] M. Inoue, K. Hiraki, I. Yokomichi, and Y. Tanaka, "Effectiveness of the particle damper with granular materials," in Proceedings of the 3rd International Conference on Integrity, Reliability and Failure, S1108-P0254, Porto, Portugal, July 2009.

[15] Y. Isao, T. Yoshito, and N. Y. So, "Particle damping with granular materials for multi-body system," in Proceedings of the 15th International Congress on Sound and Vibration (ICSV '08), Daejeon, Republic of Korea, 2008.

[16] M. Trigui, E. Foltete, M. S. Abbes, T. Fakhfakh, N. Bouhaddi, and M. Haddar, "An experimental study of a multi-particle impact damper," Proceedings of the Institution of Mechanical Engineers Part C: Journal of Mechanical Engineering Science, vol. 223, no. 9, pp. 2029-2038, 2009.

[17] W. Liu, G. R. Tomlinson, and J. A. Rongong, "The dynamic characterisation of disk geometry particle dampers," Journal of Sound and Vibration, vol. 280, no. 3-5, pp. 849-861, 2005.

[18] L. F. Bryce, M. F. Eric, and E. O. Steven, "Design methodology for particle damping," in 8th Annual International Symposium on Smart Structures and Materials: Damping and Isolation, vol. 4331 of Proceedings of SPIE, pp. 1-12, Newport Beach, Calif, USA, March 2001.

[19] K. Mao, M. Y. Wang, Z. Xu, and T. Chen, "Simulation and characterization of particle damping in transient vibrations," Journal of Vibration and Acoustics, vol. 126, no. 2, pp. 202-211, 2004.

[20] K. M. Mao, Z. W. Xu, M. Y. Wang, and T. N. Chen, "Efficient computation of particle motions in discrete element modeling of particle damping," in Proceedings of the 8th International Symposium on Plasticity and Impact Mechanics, pp. 994-1005, New Delhi, India, 2003.

[21] Z. Xu, M. Y. Wang, and T. Chen, "Particle damping for passive vibration suppression: numerical modelling and experimental investigation," Journal of Sound and Vibration, vol. 279, no. 3-5, pp. 1097-1120, 2005.

[22] C. J. Wu, W. H. Liao, and M. Y. Wang, "Modeling of granular particle damping using multiphase flow theory of gas-particle," Journal of Vibration and Acoustics, Transactions of the ASME, vol. 126, no. 2, pp. 196-201, 2004.

[23] X. Fang and J. Tang, "Granular damping in forced vibration: qualitative and quantitative analyses," Journal of Vibration and
Acoustics, Transactions of the ASME, vol. 128, no. 4, pp. 489-500, 2006.

[24] C. J. Wu, R. C. Yang, and D. Q. Wang, "An improved of granular particle damping using multiphase flow theory of gas-particle," in Proceedings of the 20th International Congress on Sound \& Vibration, Bangkok, Thailand, 2013.

[25] C. Wu, R. Yang, and D. Wang, "Prediction on vibration response of cantilever particle-damping beam based on two-phase flow theory of gas-particle," Journal of Mechanical Engineering, vol. 49, no. 10, pp. 53-61, 2013.

[26] L. S. Fan and C. Zhu, Principles of Gas-Solid Flows, Cambridge University Press, Cambridge, UK, 1998.

[27] D. G. Schaeffer, "Instability in the evolution equations describing incompressible granular flow," Journal of Differential Equations, vol. 66, no. 1, pp. 19-50, 1987.

[28] C. K. K. Lun, S. B. Savage, D. J. Jeffrey, and N. Chepurniy, "Kinetic theories for granular flow: inelastic particles in Couette flow and slightly inelastic particles in a general flowfield," Journal of Fluid Mechanics, vol. 140, no. 1, pp. 223-256, 1984.

[29] R. D. Blevins, Flow-Induced Vibration, Von Nostrand Reinhold, New York, NY, USA, 2nd edition, 1990.

[30] T. Sarpkaya, "Force on a circular cylinder in viscous oscillatory flow at low keulegan-carpenter number," Journal of Fluid Mechanics, vol. 165, pp. 61-71, 1986.

[31] N. Popplewell and S. E. Semercigil, "Performance of the bean bag impact damper for a sinusoidal external force," Journal of Sound and Vibration, vol. 133, no. 2, pp. 193-223, 1989.

[32] M. Fayed, "Handbook of powder science \& technology (2nd ed'97)," Carbohydrate Polymers, vol. 45, p. 106, 1997.

[33] P. Veeramuthuvel, K. Shankar, K. Sairajan, and R. Machavaram, "Prediction of particle damping parameters using RBF neural network," Procedia Materials Science, vol. 5, pp. 335-344, 2014.

[34] Z. Xu, M. Y. Wang, and T. Chen, "An experimental study of particle damping for beams and plates," Journal of Vibration and Acoustics, vol. 126, no. 1, pp. 141-148, 2004. 

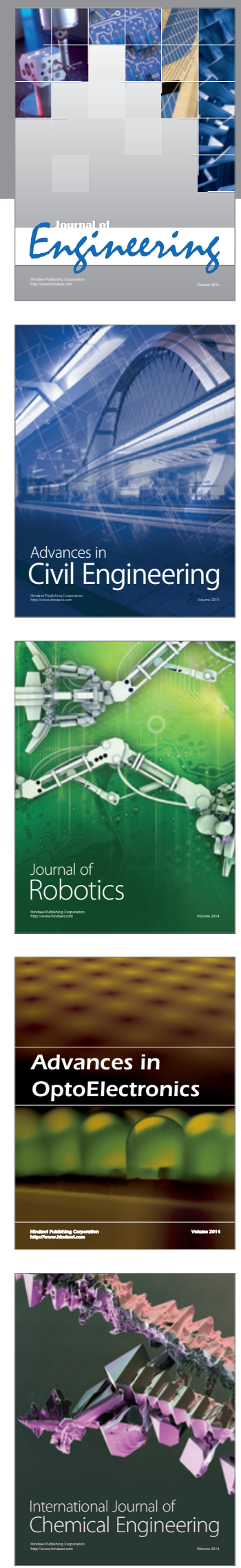

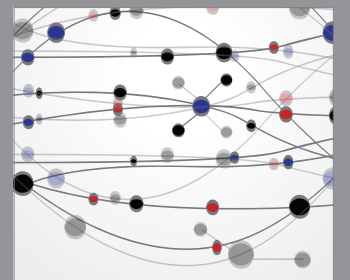

The Scientific World Journal
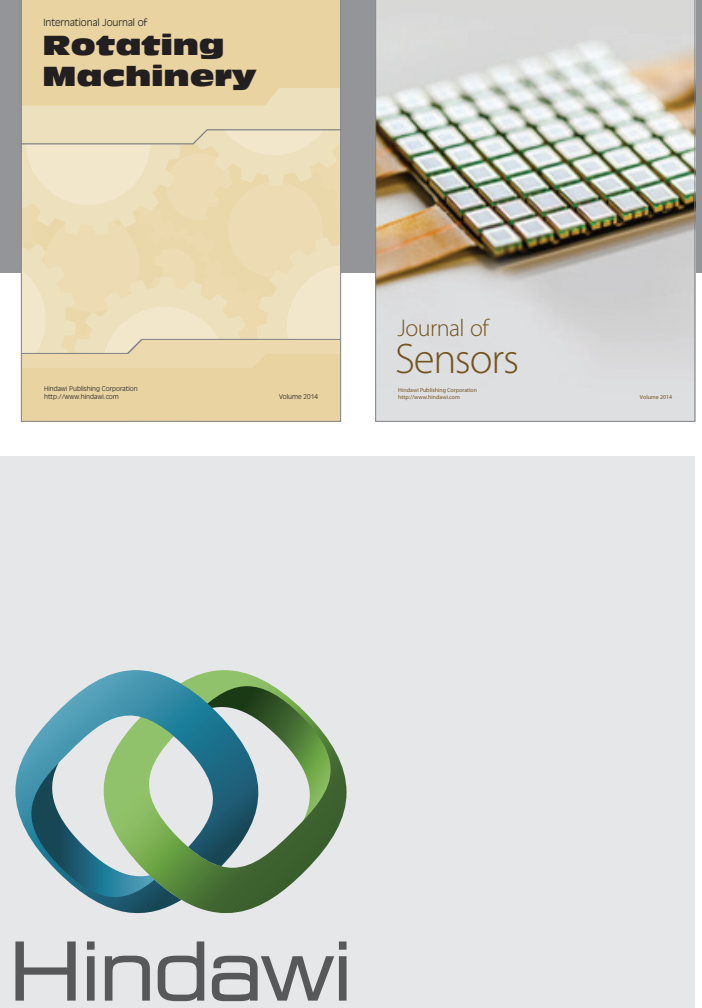

Submit your manuscripts at http://www.hindawi.com
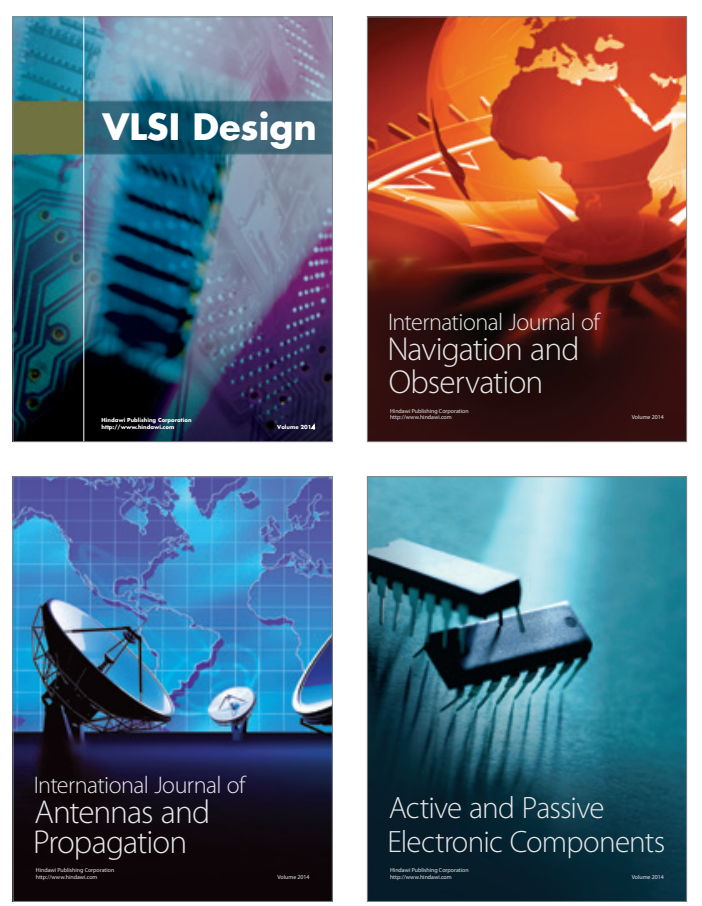
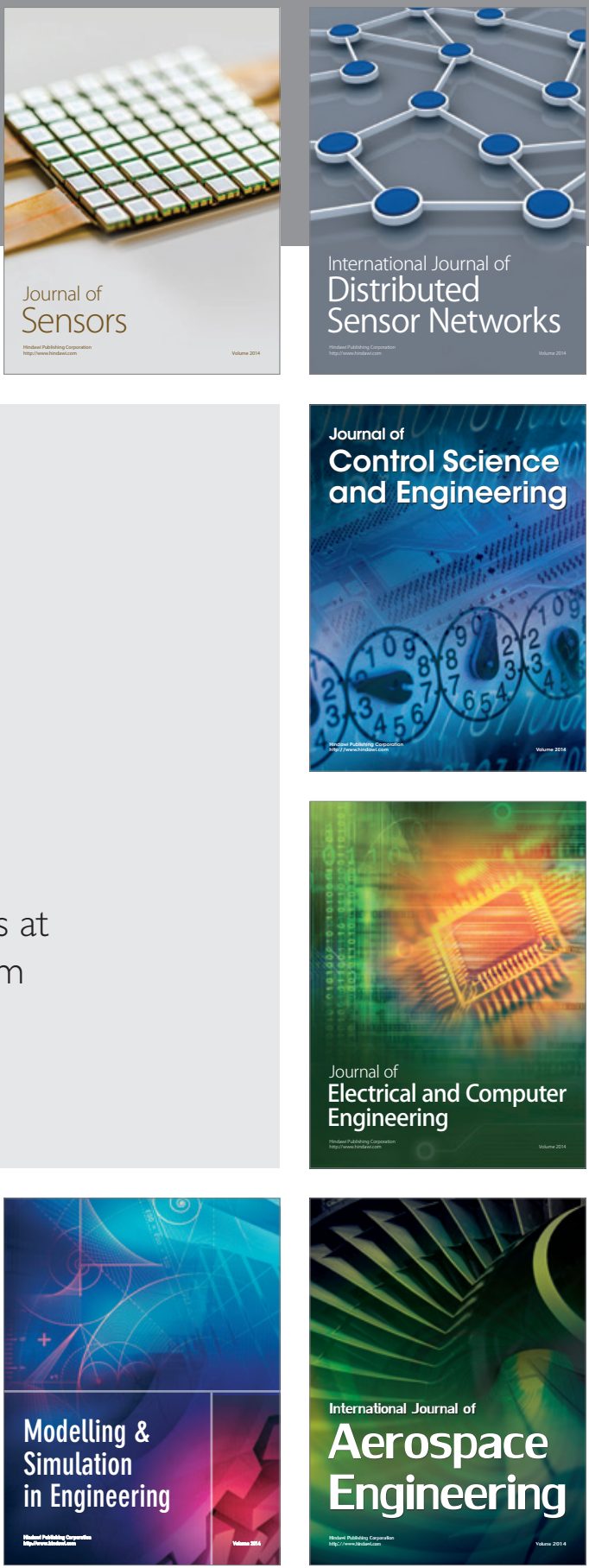

Journal of

Control Science

and Engineering
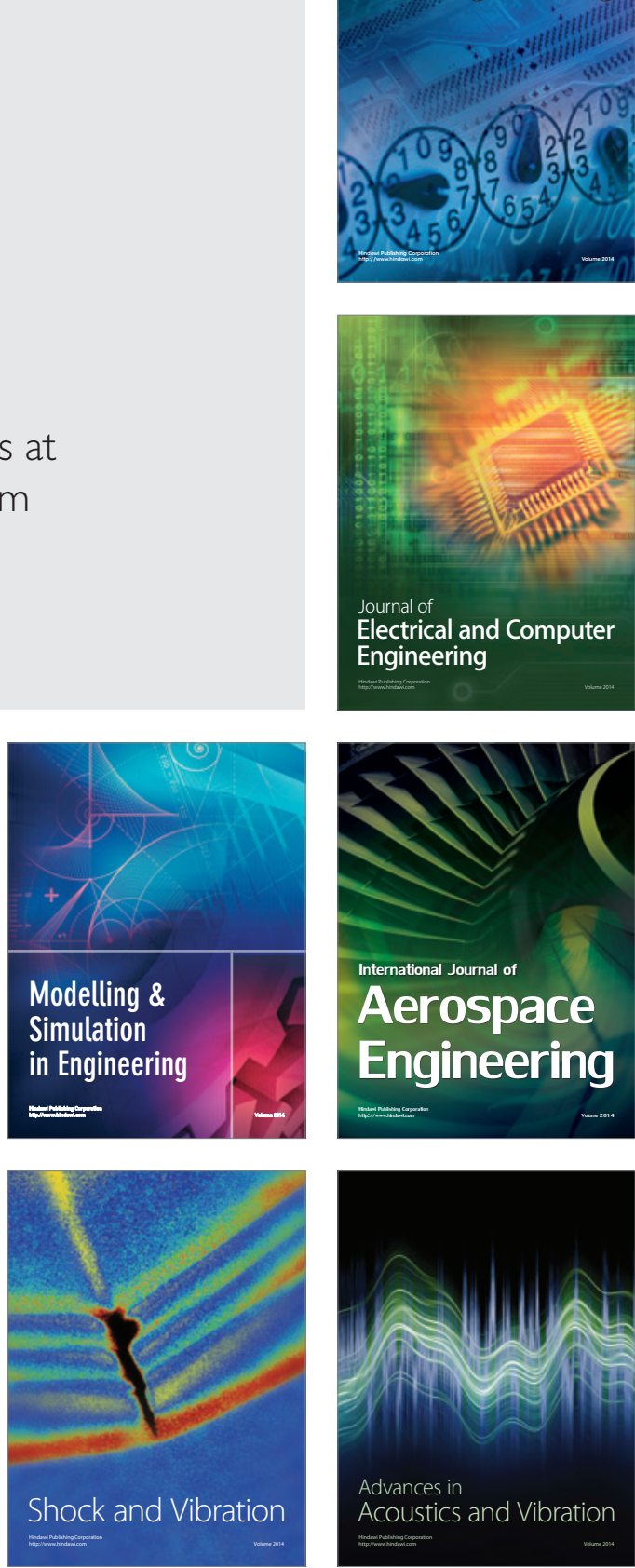